\title{
A Systematic Review of the Cost-Effectiveness of Biologics for Ulcerative Colitis
}

\author{
Ewa Stawowczyk $^{1} \cdot$ Paweł Kawalec $^{1}$ (i)
}

Published online: 19 December 2017

(c) The Author(s) 2017. This article is an open access publication

\begin{abstract}
Background Ulcerative colitis (UC) is a chronic autoimmune inflammation of the colon. The condition significantly decreases quality of life and generates a substantial economic burden for healthcare payers, patients and the society in which they live. Some patients require chronic pharmacotherapy, and access to novel biologic drugs might be crucial for long-term remission. The analyses of costeffectiveness for biologic drugs are necessary to assess their efficiency and provide the best available drugs to patients.

Objective Our aim was to collect and assess the quality of economic analyses carried out for biologic agents used in the treatment of UC, as well as to summarize evidence on the drivers of cost-effectiveness and evaluate the transferability and generalizability of conclusions.

Methods A systematic database review was conducted using MEDLINE (via PubMed), EMBASE, Cost-Effectiveness Analysis Registry and CRD0. Both authors independently reviewed the identified articles to determine their eligibility for final review. Hand searching of references in collected papers was also performed to find any relevant articles. The reporting quality of economic analyses included was evaluated by two reviewers using the
\end{abstract}

Electronic supplementary material The online version of this article (https://doi.org/10.1007/s40273-017-0601-6) contains supplementary material, which is available to authorized users.

Paweł Kawalec

pawel.kawalec@uj.edu.pl

1 Department of Drug Management, Faculty of Health Sciences, Institute of Public Health, Jagiellonian University Medical College, 20 Grzegórzecka Street, 31-531 Kraków, Poland
International Society of Pharmacoeconomics and Outcomes Research (ISPOR) Consolidated Health Economic Evaluation Reporting Standards (CHEERS) statement checklist. We reviewed the sensitivity analyses in costeffectiveness analyses to identify the variables that may have changed the conclusions of the study. Key drivers of cost-effectiveness were selected by identifying uncertain parameters that caused the highest change of the results of the analyses compared with base-case results.

Results Of the 576 identified records, 87 were excluded as duplicates and 16 studies were included in the final review; evaluations for Canada, the UK and Poland were mostly performed. The majority of the evaluations revealed were performed for infliximab (approximately 75\% of total volume); however, some assessments were also performed for adalimumab (50\%) and golimumab (31\%). Only three analyses were conducted for vedolizumab, whereas no relevant studies were found for etrolizumab and tofacitinib. The reporting quality of the included economic analyses was assessed as high, with an average score of 21 points per 24 maximum possible (range 14-23 points according to the ISPOR CHEERS statement checklist). In the case of most analyses, quality-adjusted life-years were used as a clinical outcome, and endpoints such as remission, response and mucosal healing were less common. The higher clinical effectiveness (based on response rates) of biological treatment over non-biological treatments was presented in revealed analyses. The incremental cost-utility ratios for biologics, compared with standard care, varied significantly between the studies and ranged from US $\$ 36,309$ to US\$456,979. The lowest value was obtained for infliximab and the highest for the treatment scheme including infliximab $5 \mathrm{mg} / \mathrm{kg}$ and infliximab $10 \mathrm{mg} /$ $\mathrm{kg}+$ adalimumab. The change of utility weights and clinical parameters had the most significant influence on the 
results of the analysis; the variable related to surgery was the least sensitive.

Conclusions Limited data on the cost-effectiveness of UC therapy were identified. In the majority of studies, the lack of cost-effectiveness was revealed for biologics, which was associated with their high costs. Clinical outcomes are transferable to other countries and could be generalized; however, cost inputs are country-specific and therefore limit the transferability and generalizability of conclusions. The key drivers and variables that showed the greatest effect on the analysis results were utility weights and clinical parameters.

\section{Key Points}

The majority of the identified economic evaluations related to the most commonly used agents: infliximab, adalimumab and golimumab. Most analyses were performed for Canada, the UK and Poland.

Higher clinical effectiveness (reported as response rates) of biologic drugs over reference therapies was revealed and additional clinical effect of biologics in terms of quality-adjusted life-years was presented in economic analyses. The cost-effectiveness of biological treatment, compared with standard care alone, was reported in three analyses, and, in nine studies, biologics were described as inefficient.

Infliximab was cost-effective when compared with cyclosporine or surgical treatment.

Utility weights and clinical parameters are the main key drivers of the cost-effectiveness of biologic treatments in UC.

\section{Introduction}

Ulcerative colitis (UC) is an inflammatory bowel disease characterized by the chronic inflammation of colonic mucosa. Patients with UC typically present with bloody diarrhoea, the passage of pus or mucus (or both), and abdominal cramping during bowel movements [1]. The clinical course is difficult to predict and changeable due to alternating periods of remissions and exacerbations, which may occur spontaneously or in response to external triggers (e.g. treatment changes) $[2,3]$.

The annual incidence of UC is from 0 to 19.2 per 100,000 persons in North America, and from 0.6 to 24.3 per 100,000 persons in Europe. The prevalence ranges from
37.5 to 248.6 per 100,000 persons in North America, and from 4.9 to 505 per 100,000 persons in Europe [4].

The main goal of UC pharmacotherapy is to effectively obtain the remission of symptoms, and subsequently maintain symptom-free periods. Patients affected with UC often require expensive, lifelong treatment, and sometimes surgery, both of which generate great direct costs to the healthcare payer. Conventional therapy includes corticosteroids, 5-aminosalicylates (5-ASA; 5-aminosalicylic acid) and thiopurines [3]. Biologics, which target a specific inflammatory mediator, are used in patients with moderateto-severe chronic UC who are unresponsive or intolerant to current therapy. The following biologics are currently being assessed or are already used for UC therapy: tumour necrosis factor inhibitors (infliximab, adalimumab, golimumab), integrin antagonists (vedolizumab, etrolizumab) and Janus kinase (JAK) inhibitors (tofacitinib) [5].

Clinical trials revealed that the biologics are effective in UC therapy, but also generate a heavy burden on healthcare service providers and payers $[5,6]$. The increasing prevalence and high costs of UC pharmacotherapy and surgery constitute a considerable challenge for healthcare systems and generate a need for efficient and cost-effective management of this chronic long-term disease [7]. The costeffectiveness of different treatment strategies is required to make objective and verifiable reimbursement decisions, providing patients with the best treatment regimens available.

Previous reviews on a similar topic did not provide a comprehensive assessment of the quality of cost-effectiveness analyses and the key drivers of cost-effectiveness were not identified. The objective of our study was to systematically review medical databases to collect relevant publications on the cost-effectiveness of biologic treatment in UC, and to perform a sophisticated and extensive review of the methodologies used in the economic evaluations, as well as assess the quality of the collected studies and identify the key drivers of cost-effectiveness.

\section{Materials and Methods}

\subsection{Systematic Literature Search}

A systematic literature review was performed in July 2017 in order to obtain data on the efficiency of the biologic treatments of UC. The following databases were searched: MEDLINE (via PubMed), EMBASE, the Cost-Effectiveness Analysis (CEA) Registry and Centre for Reviews and Dissemination (CRD). The following terms were used to define the population studied: ulcerative colitis, ulcerative disease, colitis ulcerosa. We focused on the biologics that are authorized for use in UC in Europe and/or in the US: 
infliximab, adalimumab, golimumab, vedolizumab, etrolizumab, and tofacitinib. The following terms were used to define the intervention: biologic, TNF, tumor necrosis factor, tumour necrosis factor, infliximab, adalimumab, golimumab, Remsima, Remicade, Inflectra, Humira, Simponi, integrin antagonist, vedolizumab, Entyvio, JAK inhibitor, Janus kinases, etrolizumab, tofacitinib, Xeljanz, Jakvinus. The following terms were also used: economic analysis, economic assessment, economic evaluation, costeffectiveness, cost efficacy, and cost-utility. A detailed search strategy is available as electronic supplementary material. The CEA Registry was searched using the term 'ulcerative colitis'. In the CRD database, we omitted terms connected with the type of analysis.

The population included was described by the Population, Intervention, Comparator, Outcome (PICO) scheme: $\mathrm{P}$-patients with moderate-to-severe UC (studies conducted in subpopulations were also included); I-biologic therapy with infliximab, adalimumab, golimumab, vedolizumab, etrolizumab or tofacitinib; $\mathrm{C}$ - not applicable, all other UC treatments were included; $\mathrm{O}-$ cost-effectiveness or cost-utility analyses.

The systematic review was independently carried out by two researchers, based on the same search strategy. The results for those searches were then compared; for disagreements, collaborative reassessment of the review results was performed to reach a final consensus.

\subsection{Study Selection}

Papers written in English were included. Neither country nor date filters were used. Original studies including primary data on the cost-effectiveness of biologics in UC, as well as reviews that led to previously undiscovered efficiency assessments, were included. Conference abstracts and posters were excluded as they did not provide the details valid for our review. The following exclusion criteria were implemented: a different intervention studied (i.e. pharmacotherapy other than biologics, surgery), different population (Crohn disease, other inflammatory bowel diseases) and different endpoints (i.e. cost assessments, clinical analyses, budget impact analyses). In this review, we included articles that fulfilled the inclusion criteria, defined within the PICO scheme, and which did not meet the exclusion criteria. During the selection of records, we used the Preferred Reporting Items for Systematic Reviews and the Meta-Analyses (PRISMA) statement.

\subsection{Extraction and Presentation of Data}

Data were extracted from the articles eligible. We used the Cochrane Handbook for Systematic Reviews of Intervention [8] to determine the terms collected: the first author's name, population, interventions, comparators, year of study, study design (assumptions and methods of the main analysis, i.e. the type of economic analysis, outcomes, perspective, country, time horizon, discount rates, cost reference date, currency, type of sensitivity analysis), results (total and incremental costs, total and incremental outcomes, incremental cost-effectiveness ratio/incremental cost-utility ratio [ICER/ICUR] value, conclusions on cost-effectiveness, results of sensitivity analysis). Two contributors discussed the discrepancies until a consensus was reached.

\subsection{Quality Assessment}

The reporting quality of the identified studies was assessed using the International Society of Pharmacoeconomics and Outcomes Research (ISPOR) Consolidated Health Economic Evaluation Reporting Standards (CHEERS) statement checklist. It does not evaluate the quality of conduct, however its objective is to ensure that the analyses are fully reported. This instrument consists of a 24-item checklist [9] verifying the presence of specific issues (e.g. perspective, comparators, time horizon) in the considered papers.

Two authors reviewed the studies and scored each question 0 (this particular aspect is not present) or 1 (this particular aspect is present). Any discrepancies were resolved by consensus.

\subsection{Synthesis of Data}

The identified studies were grouped depending on the type of biologics they investigated: infliximab, adalimumab, golimumab, vedolizumab, and other biologics. Cost-effectiveness was assessed based on conclusions presented by authors of the eligible studies.

\section{Results}

\subsection{Study Selection}

The systematic search identified 576 references. After removing duplicates, 489 remaining records were submitted for further selection based on the review of their titles and abstracts, when necessary. In the next step, we excluded 457 records for the following reasons: a different type of analysis (e.g. cost comparisons, budget impact analyses, clinical effectiveness analyses), secondary data (reviews), different intervention, different population (e.g. inflammatory bowel diseases in general, Crohn disease). Of the 32 records selected by full-text review, 16 were excluded due to secondary data (systematic reviews), ineligible publication type (conference abstracts), and different population or endpoints. The remaining 16 records were 
included in the review (Fig. 1). In our review, we found four reports for Canada, three for Poland, and two for England and Wales, as well as four for the UK. Additional evaluations were performed for The Netherlands, Iran and the US.

\subsubsection{Infliximab}

Most of the identified studies related to infliximab (75\%; $n=12$ ), two of which were cost-effectiveness analyses with response and/or remission and/or mucosal healing as the outcomes [10,11], and ten were cost-utility evaluations with quality-adjusted life-year (QALY) as an outcome. Four analyses used decision trees as an analytic technique [11-14], whereas the Markov modelling was used in the remaining evaluations. In the study by Wilson et al. [14], both the above modelling techniques were used (Table 1). Two analyses (Punekar and Hawkins [13] and Tsai et al. [15]) showed that infliximab is a cost-effective treatment option compared with standard care alone, whereas six other analyses revealed opposite conclusions [16-21], namely, the ICER/ICUR value exceeded the threshold

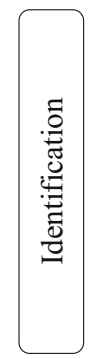

Records identified through database searching $(\mathrm{n}=574)$

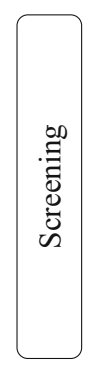

Records after duplicates removed $(\mathrm{n}=489)$

Additional records identified through other sources $(n=2)$

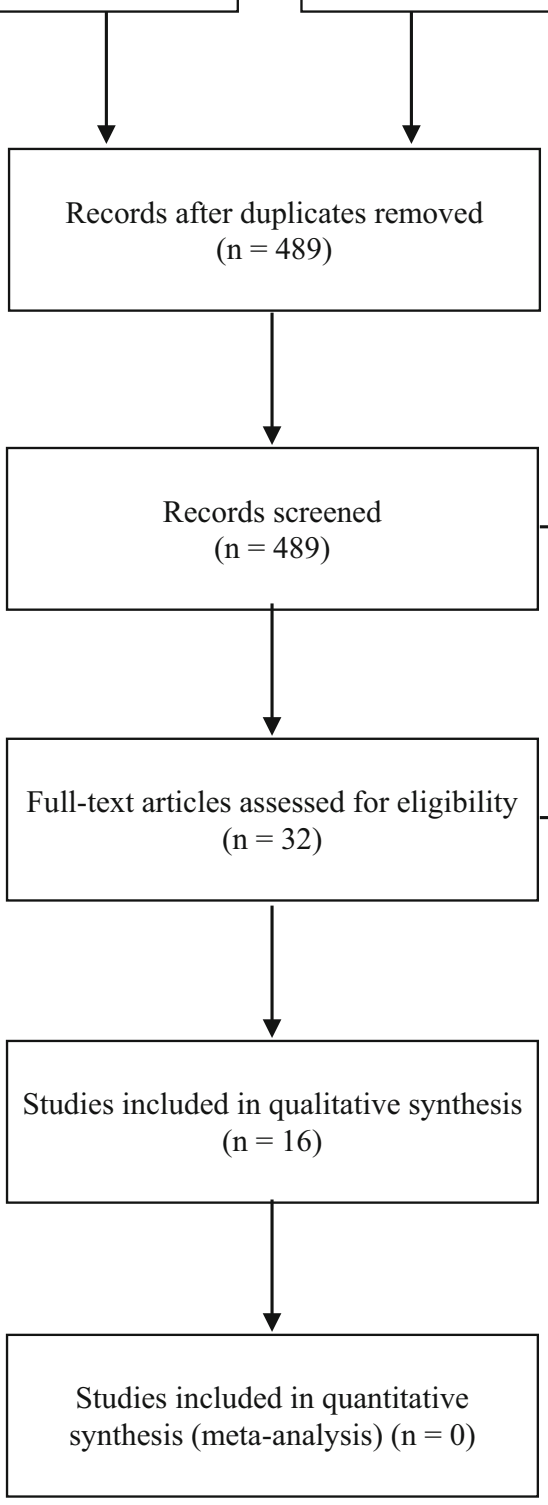

Records excluded $(n=457)$
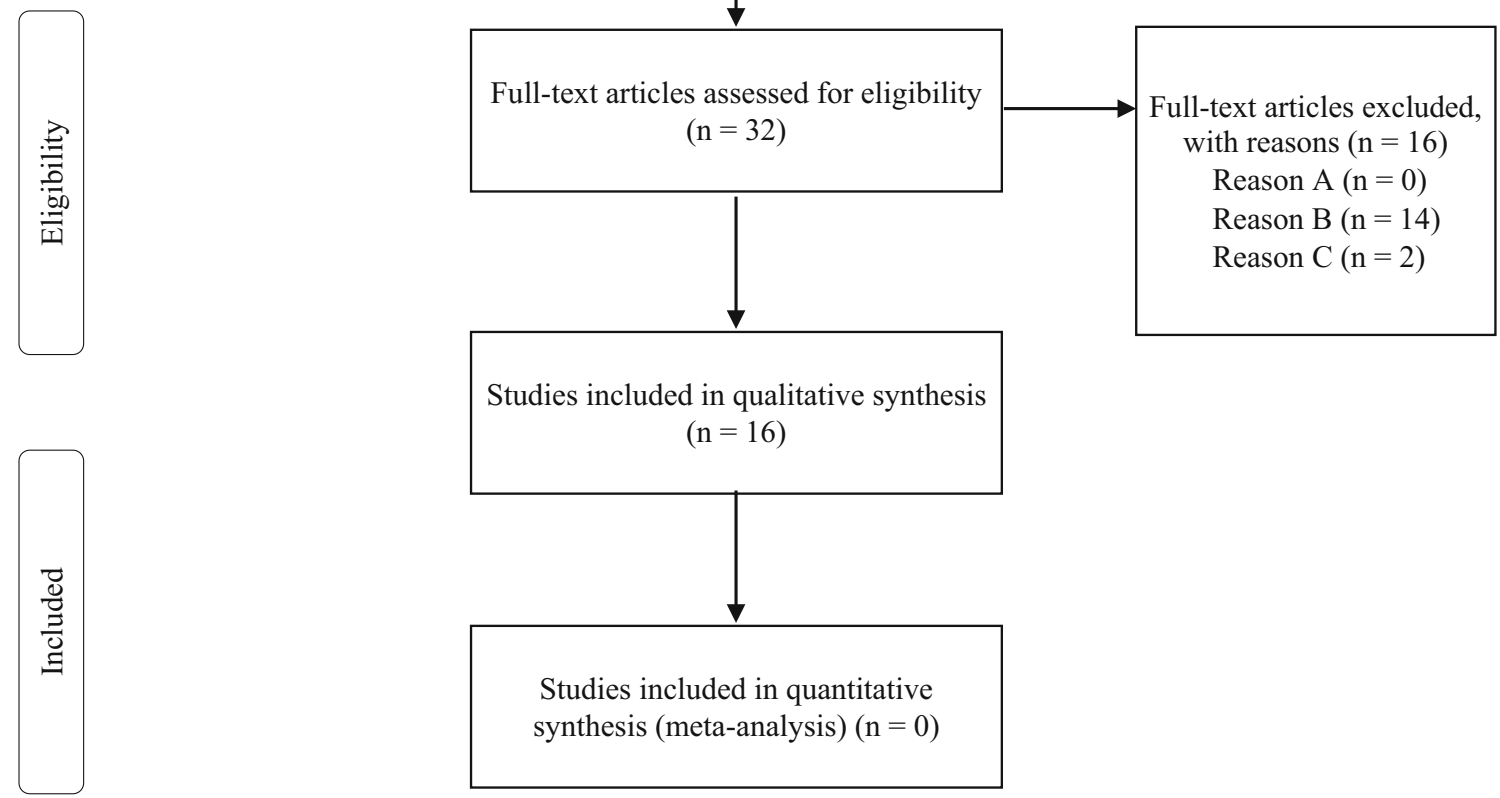

Fig. 1 PRISMA flow diagram showing the record selection process. Reason A-different intervention; Reason B-different type of study/ endpoint; Reason C-different population. PRISMA Preferred Reporting Items for Systematic Reviews and Meta-Analyses 
Table 1 Characteristics of the included studies

\begin{tabular}{|c|c|c|c|}
\hline Study, year & Population & Treatment & Study characteristics \\
\hline $\begin{array}{l}\text { Beilman } \\
\text { et al., } 2016 \\
\text { [23] }\end{array}$ & $\begin{array}{l}\text { Moderate-to-severe active UC, } \\
\text { corticosteroid-dependent and/or did not } \\
\text { respond to thiopurine therapy }\end{array}$ & $\begin{array}{l}\text { Adalimumab } 160 / 80 / \\
40 \mathrm{mg} \text {, standard care }\end{array}$ & $\begin{array}{l}\text { Type: Cost-utility analysis } \\
\text { Model: Markov } \\
\text { Horizon: } 10 \text { years } \\
\text { Outcomes: QALY } \\
\text { Discounting (costs/effects): } 5 \% / 5 \% \\
\text { Country: Canada } \\
\text { Perspective: NA } \\
\text { Reference year: NA } \\
\text { Sponsor: Supported by the Centre of Excellence for } \\
\text { Gastrointestinal Inflammation and Immunity } \\
\text { Research }\end{array}$ \\
\hline $\begin{array}{l}\text { Chaudhary } \\
\text { and Fan, } \\
2013 \text { [12] }\end{array}$ & $\begin{array}{l}\text { Sever UC adult patients hospitalized } \\
\text { with an acute exacerbation of the } \\
\text { disease }\end{array}$ & $\begin{array}{l}\text { Infliximab } 5 \mathrm{mg} / \mathrm{kg} \text {, } \\
\text { cyclosporine } 2 \mathrm{mg} / \mathrm{kg} \text {, } \\
\text { surgical intervention }\end{array}$ & $\begin{array}{l}\text { Type: Cost-utility analysis } \\
\text { Model: Decision tree } \\
\text { Horizon: } 1 \text { year } \\
\text { Outcomes: QALY } \\
\text { Discounting (costs/effects): } 4 \% / 1.5 \% \\
\text { Country: The Netherlands } \\
\text { Perspective: National payer } \\
\text { Reference year: } 2010 \\
\text { Sponsor: Merck \& Co., Inc. }\end{array}$ \\
\hline $\begin{array}{l}\text { Moradi et al., } \\
2016[19]\end{array}$ & Moderate-to-severe UC & $\begin{array}{l}\text { Infliximab } 5 \mathrm{mg} / \mathrm{kg} \text {, } \\
\text { conventional treatments }\end{array}$ & $\begin{array}{l}\text { Type: Cost-utility analysis } \\
\text { Model: Markov } \\
\text { Horizon: } 5 \text { years } \\
\text { Outcomes: QALY } \\
\text { Discounting (costs/effects): 5\%/NA } \\
\text { Country: Iran } \\
\text { Perspective: Public payer } \\
\text { Reference year: } 2014 \\
\text { Sponsor: No }\end{array}$ \\
\hline $\begin{array}{l}\text { Punekar and } \\
\text { Hawkins, } \\
2010[13]\end{array}$ & $\begin{array}{l}\text { Moderate-to-severe UC patients } \\
\text { hospitalized with an acute exacerbation } \\
\text { of the disease }\end{array}$ & $\begin{array}{l}\text { Infliximab } 5 \mathrm{mg} / \mathrm{kg} \text {, } \\
\text { cyclosporine } 4 \mathrm{mg} / \mathrm{kg} \text {, } \\
\text { surgery, standard care }\end{array}$ & $\begin{array}{l}\text { Type: Cost-utility analysis } \\
\text { Model: Decision tree } \\
\text { Horizon: } 1 \text { year } \\
\text { Outcomes: QALY } \\
\text { Discounting (costs/effects): } 3.5 \% / 3.5 \% \\
\text { Country: England, Wales } \\
\text { Perspective: Public payer } \\
\text { Reference year: 2006-2007 } \\
\text { Sponsor: Schering-Plough Ltd }\end{array}$ \\
\hline $\begin{array}{l}\text { Stawowczyk } \\
\text { et al., } 2016 \\
\text { [22] }\end{array}$ & Moderate-to-severe UC & $\begin{array}{l}\text { Adalimumab } 160 / 80 / \\
40 \mathrm{mg} \text {, standard care }\end{array}$ & $\begin{array}{l}\text { Type: Cost-utility analysis } \\
\text { Model: Markov } \\
\text { Horizon: } 30 \text { years } \\
\text { Outcomes: QALY } \\
\text { Discounting (costs/effects): } 5 \% / 3.5 \% \\
\text { Country: Poland } \\
\text { Perspective: Public payer, social } \\
\text { Reference year: } 2015 \\
\text { Sponsor: No }\end{array}$ \\
\hline
\end{tabular}


Table 1 continued

\begin{tabular}{|c|c|c|c|}
\hline Study, year & Population & Treatment & Study characteristics \\
\hline $\begin{array}{l}\text { Stawowczyk } \\
\text { et al., } 2016 \\
{[24]}\end{array}$ & Moderate-to-severe UC & $\begin{array}{l}\text { Golimumab 200/100/ } \\
50 \mathrm{mg} \text {, standard care }\end{array}$ & $\begin{array}{l}\text { Type: Cost-utility analysis } \\
\text { Model: Markov } \\
\text { Horizon: } 30 \text { years } \\
\text { Outcomes: QALY } \\
\text { Discounting (costs/effects): } 5 \% / 3.5 \% \\
\text { Country: Poland } \\
\text { Perspective: Public payer, social } \\
\text { Reference year: } 2015 \\
\text { Sponsor: No }\end{array}$ \\
\hline $\begin{array}{l}\text { Stawowczyk } \\
\text { et al., } 2016 \\
{[21]}\end{array}$ & Moderate-to-severe UC & $\begin{array}{l}\text { Infliximab } 5 \mathrm{mg} / \mathrm{kg} \text {, } \\
\text { standard care }\end{array}$ & $\begin{array}{l}\text { Type: Cost-utility analysis } \\
\text { Model: Markov } \\
\text { Horizon: } 30 \text { years } \\
\text { Outcomes: QALY } \\
\text { Discounting (costs/effects): 5\%/3.5\% } \\
\text { Country: Poland } \\
\text { Perspective: Public payer } \\
\text { Reference year: } 2015 \\
\text { Sponsor: No }\end{array}$ \\
\hline $\begin{array}{l}\text { Tappenden } \\
\text { et al., } 2016 \\
{[17]}\end{array}$ & $\begin{array}{l}\text { Moderate-to-severe UC in patients for } \\
\text { whom at least one prior therapy has } \\
\text { failed }\end{array}$ & $\begin{array}{l}\text { Infliximab } 5 \mathrm{mg} / \mathrm{kg} \text {, } \\
\text { adalimumab } 160 / 80 / \\
40 \mathrm{mg} \text {, golimumab } 200 \mathrm{~g} / \\
100 / 100 \mathrm{mg}(50 \mathrm{mg}), \\
\text { conventional non-biologic } \\
\text { therapy, elective surgery }\end{array}$ & $\begin{array}{l}\text { Type: Cost-utility analysis } \\
\text { Model: Markov } \\
\text { Horizon: } 60 \text { years } \\
\text { Outcomes: QALY } \\
\text { Discounting (costs/effects): } 3.5 \% / 3.5 \% \\
\text { Country: UK } \\
\text { Perspective: Public payer, Personal Social Services } \\
\text { Reference year: } 2013 / 2014 \\
\text { Sponsor: National Institute for Health Research Health } \\
\text { Technology Assessment Programme }\end{array}$ \\
\hline $\begin{array}{l}\text { Toor et al., } \\
2015[10]\end{array}$ & Moderate-to-severe UC & $\begin{array}{l}\text { Infliximab } 5 \mathrm{mg} / \mathrm{kg} \text {, } \\
\text { adalimumab } 40 \mathrm{mg} \text {, } \\
\text { golimumab } 50 / 100 \mathrm{mg} \text {, } \\
\text { conventional therapy }\end{array}$ & $\begin{array}{l}\text { Type: Cost-effectiveness analysis } \\
\text { Model: Markov } \\
\text { Horizon: } 1 \text { year } \\
\text { Outcomes: Remission, response } \\
\text { Discounting (costs/effects): - } \\
\text { Country: Canada } \\
\text { Perspective: Public payer } \\
\text { Reference year: } 2013 \\
\text { Sponsor: Janssen Inc. Canada }\end{array}$ \\
\hline $\begin{array}{l}\text { Tsai et al., } \\
2008[15]\end{array}$ & Moderate-to-severe UC & $\begin{array}{l}\text { Infliximab } 5 \mathrm{mg} / \mathrm{kg} \text {, } \\
\text { standard care }\end{array}$ & $\begin{array}{l}\text { Type: Cost-utility analysis } \\
\text { Model: Markov } \\
\text { Horizon: } 10 \text { years } \\
\text { Outcomes: QALY } \\
\text { Discounting (costs/effects): } 3.5 \% / 3.5 \% \\
\text { Country: England, Wales } \\
\text { Perspective: Public payer } \\
\text { Reference year: } 2006 / 2007 \\
\text { Sponsor: Schering-Plough Ltd }\end{array}$ \\
\hline
\end{tabular}


Table 1 continued

\begin{tabular}{|c|c|c|c|}
\hline Study, year & Population & Treatment & Study characteristics \\
\hline $\begin{array}{l}\text { Ung et al., } \\
2014[20]\end{array}$ & Moderate-to-severe UC & $\begin{array}{l}\text { Infliximab } 5 \mathrm{mg} / \mathrm{kg} \text {, } \\
\text { standard care }\end{array}$ & $\begin{array}{l}\text { Type: Cost-utility analysis } \\
\text { Model: Markov } \\
\text { Horizon: } 10 \text { years } \\
\text { Outcomes: QALY } \\
\text { Discounting (costs/effects): } 5 \% / 5 \% \\
\text { Country: Canada } \\
\text { Perspective: Health system } \\
\text { Reference year: } 2013 \\
\text { Sponsor: Supported by the Centre of Excellence for } \\
\text { Gastrointestinal Inflammation and Immunity } \\
\text { Research and the Alberta Innovates }\end{array}$ \\
\hline $\begin{array}{l}\text { Wilson et al., } \\
2017 \text { [14] }\end{array}$ & Moderate-to-severe UC & $\begin{array}{l}\text { Vedolizumab } 300 \mathrm{mg} \text {, } \\
\text { infliximab } 5 \mathrm{mg} / \mathrm{kg} \text {, } \\
\text { adalimumab } 160 / 80 / \\
40 \mathrm{mg} \text {, golimumab } \\
100 / 50 / 50 \mathrm{mg}, \\
\text { conventional therapy }\end{array}$ & $\begin{array}{l}\text { Type: Cost-utility analysis } \\
\text { Model: Decision tree/Markov } \\
\text { Horizon: Lifetime } \\
\text { Outcomes: QALY } \\
\text { Discounting (costs/effects): } 3.5 \% / 3.5 \% \\
\text { Country: UK } \\
\text { Perspective: Public payer, Personal Social Services } \\
\text { Reference year: } 2012 / 2013 \\
\text { Sponsor: Takeda Pharmaceuticals, AG }\end{array}$ \\
\hline $\begin{array}{l}\text { Xie et al., } \\
2009[18]\end{array}$ & Moderate-to-severe refractory UC & $\begin{array}{l}\text { Infliximab 5/10 mg, } \\
\text { adalimumab 160/80/ } \\
40 \mathrm{mg} \text {, usual care }\end{array}$ & $\begin{array}{l}\text { Type: Cost-utility analysis } \\
\text { Model: Markov } \\
\text { Horizon: } 5 \text { years } \\
\text { Outcomes: QALY } \\
\text { Discounting (costs/effects): } 5 \% 15 \% \\
\text { Country: Canada } \\
\text { Perspective: Public payer } \\
\text { Reference year: } 2008 \\
\text { Sponsor: No }\end{array}$ \\
\hline $\begin{array}{l}\text { Yokomizo } \\
\text { et al., } 2016 \\
{[11]}\end{array}$ & Moderate-to-severe UC & $\begin{array}{l}\text { Infliximab 5/10 mg, } \\
\text { adalimumab 160/80/ } \\
40 \mathrm{mg} \text {, vedolizumab } 300 \\
\mathrm{mg}\end{array}$ & $\begin{array}{l}\text { Type: Cost-effectiveness analysis } \\
\text { Model: Decision tree } \\
\text { Horizon: } 1 \text { year } \\
\text { Outcomes: Remission, mucosal healing } \\
\text { Discounting (costs/effects): - } \\
\text { Country: US } \\
\text { Perspective: Third party's } \\
\text { Reference year: } 2014 \\
\text { Sponsor: National Institute of Diabetes and Digestive } \\
\text { and Kidney Diseases }\end{array}$ \\
\hline $\begin{array}{l}\text { Archer et al., } \\
2016[16]\end{array}$ & $\begin{array}{l}\text { Moderate-to-severe UC, after the failure } \\
\text { of conventional treatment }\end{array}$ & $\begin{array}{l}\text { Infliximab } 5 \mathrm{mg} / \mathrm{kg} \text {, } \\
\text { adalimumab } 160 / 80 / \\
40 \mathrm{mg}, \text { golimumab } \\
200 / 100 / 100 \mathrm{mg}(50 \mathrm{mg}) \\
\text { conventional therapy, } \\
\text { colectomy }\end{array}$ & $\begin{array}{l}\text { Type: Cost-utility analysis } \\
\text { Model: Markov } \\
\text { Horizon: Lifetime } \\
\text { Outcomes: QALY } \\
\text { Discounting (costs/effects): } 3.5 \% / 3.5 \% \\
\text { Country: UK } \\
\text { Perspective: Public payer, Personal Social Services } \\
\text { Reference year: } 2013 \\
\text { Sponsor: National Institute for Health Research }\end{array}$ \\
\hline
\end{tabular}


Table 1 continued

\begin{tabular}{clll}
\hline Study, year & Population & Treatment & Study characteristics \\
\hline $\begin{array}{c}\text { Essat et al., } \\
2016[25]^{\text {a }}\end{array}$ & Moderate-to-severe UC & $\begin{array}{c}\text { Vedolizumab, conventional } \\
\text { therapy, surgery, }\end{array}$ & Type: Cost-utility analysis \\
& infliximab, adalimumab, & Model: Markov, decision-tree & Horizon: Lifetime \\
& golimumab & Outcomes: QALY \\
& & Discounting (costs/effects): NA \\
& & Country: UK \\
& & Perspective: Public payer \\
& & Reference year: NA \\
& & Sponsor: Evidence Review Group
\end{tabular}

$U C$ ulcerative colitis, $Q A L Y$ quality-adjusted life-years, $N A$ not available, $E R G$ Evidence Review Group

${ }^{a}$ Methods and results of the ERG's own calculations were included

value in a particular country. In the study by Chaudhary and Fan [12], infliximab was shown to be cost-effective when compared with cyclosporine therapy and surgical intervention. Toor et al. [10] concluded that infliximab had the highest clinical efficacy (presented as response and remission), but also generated high costs compared with adalimumab and golimumab. In comparison with adalimumab and vedolizumab, infliximab turned out to be the most cost-effective treatment option when remission and mucosal healing were taken into account [11]. In the study by Archer et al. [16], infliximab was expected to be dominated by adalimumab (Table 2).

\subsubsection{Adalimumab}

Adalimumab was assessed in eight studies, six of which were cost-utility analyses displaying QALY as an outcome $[14,16,17,18,22,23]$. The Markov modelling was used in seven studies, decision trees in two studies [11, 14], and, in the case of Wilson et al. [14], both analytic techniques (Markov modelling and the decision tree) were used (Table 1). Four studies showed that adalimumab is not a cost-effective treatment option when compared with standard care alone [16-18, 22], and only one study reported the opposite conclusion [23]. Toor et al. [10] concluded that adalimumab produced higher cost per remission and cost per response, when compared with standard care, than other biologics, i.e. infliximab and golimumab. In the study by Yokomizo et al. [11], adalimumab was dominated (proving less effective and more costly) by infliximab, when mucosal healing was included as an outcome (Table 2).

\subsubsection{Golimumab}

Golimumab was included as a treatment option in UC in five studies, four of which were cost-utility analyses $[14,16,17,24]$, and one included remission and response as the outcomes [10]. All the studies used the Markov modelling and one study additionally used a decision tree [14] (Table 1). Stawowczyk et al. [24], Tappenden et al. [17] and Archer et al. [16] concluded that golimumab is not a cost-effective treatment option when compared with standard care alone. In the study by Toor et al. [10], golimumab had the lowest cost of 1 additional year of remission and response compared with standard care, among other biologics included, i.e. infliximab and adalimumab. Wilson et al. [14] showed that golimumab was dominated by vedolizumab (Table 2).

\subsubsection{Vedolizumab}

Only three studies assessed the cost-effectiveness of vedolizumab in the treatment of UC; one used a decision tree [11] and two used both the decision tree and Markov modelling $[14,25]$. Wilson et al. [14] compared vedolizumab with other biologics used in UC, i.e. infliximab, adalimumab and golimumab, and concluded that it is a cost-effective treatment option (Table 1). Vedolizumab generated the highest outcomes (QALYs) and was cheaper than infliximab and golimumab (therefore it dominated both comparators). Only adalimumab proved to be cheaper than vedolizumab, but also less effective-the ICER for vedolizumab compared with adalimumab did not exceed the threshold value. Yokomizo et al. [11] included mucosal healing as an outcome and showed that vedolizumab was dominated by infliximab. Essat et al. [25] described the results of Evidence Review Group (ERG) assessment of vedolizumab. Based on the ERG's own calculations, it was shown that vedolizumab is expected to be dominated by surgery (Table 2).

\subsection{Quality Assessment}

The results of quality assessment with the ISPOR CHEERS statement checklist are shown in Table 3 . The study by 
Table 2 Results of the included studies

\begin{tabular}{|c|c|c|c|c|}
\hline Study, year & Total costs & Total outcomes & ICER & Authors' conclusions \\
\hline $\begin{array}{l}\text { Beilman et al., } \\
2016[23]\end{array}$ & $\begin{array}{l}\mathrm{SC}=\mathrm{US} \$ 97,000 \\
\mathrm{ADA}=\mathrm{US} \$ 107,000\end{array}$ & $\begin{array}{l}\mathrm{SC}=3.154 \\
\mathrm{ADA}=3.321\end{array}$ & $\begin{array}{l}\text { Per QALYG: } \\
\text { ADA vs. SC }=\mathrm{US} \$ 59,000\end{array}$ & $\begin{array}{l}\text { ADA is cost-effective } \\
\text { compared with SC }\end{array}$ \\
\hline $\begin{array}{l}\text { Chaudhary and } \\
\text { Fan, } 2013[12]\end{array}$ & $\begin{array}{l}\mathrm{IFX}=€ 17,062 \\
\mathrm{CSP}=€ 14,784 \\
\mathrm{~S}=€ 13,979\end{array}$ & $\begin{array}{l}\mathrm{IFX}=0.80 \\
\mathrm{CSP}=0.70 \\
\mathrm{~S}=0.58\end{array}$ & $\begin{array}{l}\text { Per QALYG: } \\
\text { IFX vs. CSP }=€ 24,277 \\
\text { IFX vs. } S=€ 14,639\end{array}$ & $\begin{array}{l}\text { IFX is cost-effective compared } \\
\text { with CSP and S }\end{array}$ \\
\hline $\begin{array}{l}\text { Moradi et al., } \\
2016[19]\end{array}$ & $\begin{array}{l}\mathrm{IFX}=\mathrm{US} \$ 77,138 \\
\mathrm{SC}=\mathrm{US} \$ 985\end{array}$ & $\begin{array}{l}\mathrm{IFX}=3.56 \\
\mathrm{SC}=3.24\end{array}$ & $\begin{array}{l}\text { Per QALYG: } \\
\text { IFX vs. SC = US } \$ 240,903\end{array}$ & $\begin{array}{l}\text { IFX is not cost-effective } \\
\text { compared with SC }\end{array}$ \\
\hline $\begin{array}{l}\text { Punekar and } \\
\text { Hawkins, } 2010 \\
\text { [13] }\end{array}$ & $\begin{array}{l}\mathrm{S}=£ 17,067 \\
\mathrm{CSP}=£ 18,122 \\
\mathrm{SC}=£ 18,524 \\
\mathrm{IFX}=£ 19,847\end{array}$ & $\begin{array}{l}S=0.58 \\
C S P=0.70 \\
S C=0.68 \\
I F X=0.80\end{array}$ & $\begin{array}{l}\text { Per QALYG: } \\
\text { IFX vs. CSP }=£ 19,545\end{array}$ & $\begin{array}{l}\text { IFX is cost-effective compared } \\
\text { with CSP, S, SC }\end{array}$ \\
\hline $\begin{array}{l}\text { Stawowczyk } \\
\text { et al., } 2016 \text { [22] }\end{array}$ & $\begin{array}{l}\text { Public payer: } \\
\text { ADA }=€ 20,598 \\
\text { SC }=€ 9950 \\
\text { Social: } \\
\text { ADA }=€ 93,765 \\
S C=€ 83,770\end{array}$ & $\begin{array}{l}\mathrm{ADA}=15.204 \\
\mathrm{SC}=15.064\end{array}$ & $\begin{array}{l}\text { Per QALYG: } \\
\text { Public payer: } \\
\text { ADA vs. } \mathrm{SC}=€ 76,120 \\
\text { Social: } \\
\text { ADA vs. } \mathrm{SC}=€ 71,457\end{array}$ & $\begin{array}{l}\text { ADA is not cost-effective } \\
\text { compared with SC }\end{array}$ \\
\hline $\begin{array}{l}\text { Stawowczyk } \\
\text { et al., } 2016[24]\end{array}$ & $\begin{array}{l}\text { Public payer: } \\
\text { GOL = PLN93,321 } \\
\text { SC = PLN45,502 } \\
\text { Social: } \\
\text { GOL = PLN302,848 } \\
\text { SC }=\text { PLN257,092 }\end{array}$ & $\begin{array}{l}\mathrm{GOL}=19.241 \\
\mathrm{SC}=19.118\end{array}$ & $\begin{array}{l}\text { Per QALYG: } \\
\text { Public payer: } \\
\text { GOL vs. SC }=391,252 \text { PLN } \\
\text { Social: } \\
\text { GOL vs. SC }=374,377 \text { PLN }\end{array}$ & $\begin{array}{l}\text { GOL is not cost-effective } \\
\text { compared with SC }\end{array}$ \\
\hline $\begin{array}{l}\text { Stawowczyk } \\
\text { et al., } 2016 \text { [21] }\end{array}$ & $\begin{array}{l}\text { IFX }=\text { PLN99,522 } \\
S C=\text { PLN29,642 }\end{array}$ & $\begin{array}{l}\mathrm{IFX}=14.296 \\
\mathrm{SC}=14.123\end{array}$ & $\begin{array}{l}\text { Per QALYG: } \\
\text { IFX vs. SC = PLN } 402,420\end{array}$ & $\begin{array}{l}\text { IFX is not cost-effective } \\
\text { compared with SC }\end{array}$ \\
\hline $\begin{array}{l}\text { Tappenden et al., } \\
2016 \text { [17] }\end{array}$ & $\begin{array}{l}\mathrm{S}=£ 56,268 \\
\mathrm{ADA}=£ 91,222 \\
\mathrm{IFX}=£ 96,595 \\
\mathrm{GOL}=£ 90,087 \\
\mathrm{SC}=£ 73,620\end{array}$ & $\begin{array}{l}\mathrm{S}=14.71 \\
\mathrm{ADA}=10.82 \\
\mathrm{IFX}=10.81 \\
\mathrm{GOL}=10.63 \\
\mathrm{SC}=10.47\end{array}$ & $\begin{array}{l}\text { Per QALYG: } \\
\text { S vs. ADA/IFX/GOL/SC-S dominated all } \\
\text { therapies } \\
\text { ADA vs. } \mathrm{SC}=£ 50,278 \\
\text { IFX vs. } \mathrm{SC}=£ 67,574^{\mathrm{a}} \\
\text { GOL vs. } \mathrm{SC}=£ 102,919^{\mathrm{a}}\end{array}$ & $\begin{array}{l}\text { Colectomy dominated all } \\
\text { medical treatment. } \\
\text { ADA, IFX }^{\mathrm{a}}, \mathrm{GOL}^{\mathrm{a}} \text { are not cost- } \\
\text { effective compared with SC }\end{array}$ \\
\hline $\begin{array}{l}\text { Toor et al., } 2015 \\
\text { [10] }\end{array}$ & NA & NA & $\begin{array}{l}\text { Per remission: } \\
\text { GOL } 50 \text { vs. } \mathrm{SC}=\mathrm{CAN} \$ 1048 \\
\text { GOL } 100 \text { vs. } \mathrm{SC}=\mathrm{CAN} \$ 935 \\
\text { IFX vs. } \mathrm{SC}=\mathrm{CAN} \$ 1975 \\
\text { ADA vs. SC }=\mathrm{CAN} \$ 7430 \\
\text { IFX vs. GOL } 100=\mathrm{CAN} \$ 14,659 \\
\text { ADA vs. GOL } 100=-\mathrm{CAN} \$ 3324 \\
\text { Per response: } \\
\text { GOL } 50 \text { vs. SC }=\mathrm{CAN} \$ 770 \\
\text { GOL } 100 \text { vs. SC }=\mathrm{CAN} \$ 701 \\
\text { IFX vs. SC }=\mathrm{CAN} \$ 1311 \\
\text { ADA vs. SC }=\mathrm{CAN} \$ 2361 \\
\text { IFX vs. GOL } 100=\mathrm{CAN} \$ 4753 \\
\text { ADA vs. GOL } 100=-\mathrm{CAN} \$ 4019\end{array}$ & $\begin{array}{l}\text { GOL } 100 \text { and GOL } 50 \text { have the } \\
\text { lowest cost of additional } \\
1 \text { year of remission and } \\
\text { response; IFX has the highest } \\
\text { efficacy, but also high costs, } \\
\text { ADA produced the highest } \\
\text { cost/remission and response }\end{array}$ \\
\hline
\end{tabular}


Table 2 continued

\begin{tabular}{|c|c|c|c|c|}
\hline Study, year & Total costs & Total outcomes & ICER & Authors' conclusions \\
\hline $\begin{array}{l}\text { Tsai et al., } 2008 \\
\text { [15] }\end{array}$ & $\begin{array}{l}\text { Responders only: } \\
\text { IFX }=£ 66,460 \\
\text { SC }=£ 45,798 \\
\text { Remission only: } \\
\text { IFX }=£ 53,874 \\
\text { SC }=£ 46,259\end{array}$ & $\begin{array}{l}\text { Responders } \\
\text { only: } \\
\text { IFX }=4.591 \\
\mathrm{SC}=3.838 \\
\text { Remission only: } \\
\text { IFX }=4.154 \\
\mathrm{SC}=3.767\end{array}$ & $\begin{array}{l}\text { Per QALYG: } \\
\text { Responders only: } \\
\text { IFX vs. SC = } 27,424 \\
\text { Remission only: } \\
\text { IFX vs. SC = } 19,696\end{array}$ & $\begin{array}{l}\text { IFX is cost-effective compared } \\
\text { with SC }\end{array}$ \\
\hline $\begin{array}{l}\text { Ung et al., } 2014^{\mathrm{b}} \\
\text { [20] }\end{array}$ & $\begin{array}{l}\mathrm{SC}=\mathrm{US} \$ 86,000 \\
\mathrm{IFX}=\mathrm{US} \$ 98,000\end{array}$ & $\begin{array}{l}\mathrm{SC}=3.204 \\
\mathrm{IFX}=3.284\end{array}$ & Per QALYG: IFX vs. SC $=$ US $\$ 152,000$ & $\begin{array}{l}\text { IFX is not cost-effective } \\
\text { compared with SC }\end{array}$ \\
\hline $\begin{array}{l}\text { Wilson et al., } \\
2017 \text { [14] }\end{array}$ & $\begin{array}{l}\mathrm{VED}=£ 199,431 \\
\mathrm{IFX}=£ 206,066 \\
\mathrm{ADA}=£ 194,765 \\
\mathrm{GOL}=£ 200,018\end{array}$ & $\begin{array}{l}\text { VED }=14.077 \\
\mathrm{IFX}=13.788 \\
\mathrm{ADA}=13.872 \\
\mathrm{GOL}=13.809\end{array}$ & $\begin{array}{l}\text { Per QALYG: } \\
\text { VED vs. IFX_VED dominated IFX } \\
\text { VED vs. ADA = } 22,775 \\
\text { VED vs. GOL_-VED dominated GOL }\end{array}$ & $\begin{array}{l}\text { VED is cost-effective } \\
\text { compared with IFX, ADA, } \\
\text { GOL }\end{array}$ \\
\hline $\begin{array}{l}\text { Xie et al., } 2009 \\
\text { [18] }\end{array}$ & $\begin{array}{l}\mathrm{SC}=\mathrm{CAN} \$ 24,268 \\
\mathrm{IFX} \\
\text { ADA }=\text { CAN } \$ 82,756 \\
\mathrm{IFX} 5 \mathrm{IFX} 10 \\
\text { ADA = CAN } \$ 101,272\end{array}$ & $\begin{array}{l}\mathrm{SC}=2.015 \\
\mathrm{IFX} \\
\mathrm{ADA}=2.178 \\
\mathrm{IFX} 5 \mathrm{IFX} 10 \\
\text { ADA }=2.149\end{array}$ & $\begin{array}{l}\text { Per QALYG: } \\
\text { IFX ADA vs. SC = CAN } \$ 358,088 \\
\text { IFX } 5 \text { IFX } 10 \text { ADA vs. } \\
\text { SC = CAN } \$ 575,540\end{array}$ & $\begin{array}{l}\text { Anti-TNF } \alpha \text { therapies are not } \\
\text { cost-effective compared with } \\
\text { SC }\end{array}$ \\
\hline $\begin{array}{l}\text { Yokomizo et al., } \\
2016[11]\end{array}$ & $\begin{array}{l}\text { IFX } 5=\text { US } \$ 99,171 \\
\text { IFX } 10=\text { US } \$ 123,653 \\
\text { ADA }=\text { US } \$ 316,378 \\
\text { VED }=\text { US } \$ 301,969\end{array}$ & NA & $\begin{array}{l}\text { Per mucosal healing: } \\
\text { IFX } 5 \text { vs. IFX } 10=\text { US } \$ 1,243,310 \\
\text { IFX } 5 \text { vs. ADA-IFX dominated ADA } \\
\text { IFX } 5 \text { vs. VED-IFX dominated VED }\end{array}$ & $\begin{array}{l}\text { IFX is the most cost-effective } \\
\text { treatment option }\end{array}$ \\
\hline $\begin{array}{l}\text { Archer et al., } \\
2016[16]\end{array}$ & $\begin{array}{l}\mathrm{S}=£ 56,267.73 \\
\mathrm{ADA}=£ 91,221.71 \\
\mathrm{IFX}=£ 96,594.62 \\
\mathrm{GOL}=£ 90,086.69 \\
\mathrm{SC}=£ 73,619.77\end{array}$ & $\begin{array}{l}\mathrm{S}=14.71 \\
\mathrm{ADA}=10.82 \\
\mathrm{IFX}=10.81 \\
\mathrm{GOL}=10.63 \\
\mathrm{SC}=10.47\end{array}$ & $\begin{array}{l}\text { IFX vs. } \mathrm{ADA}-\mathrm{ADA} \text { dominated IFX } \\
\text { ADA vs. } \mathrm{SC}=£ 50,278 \\
\text { IFX vs. } \mathrm{SC}=£ 67,573^{\mathrm{a}} \\
\text { GOL vs. } \mathrm{SC}=£ 102,918^{\mathrm{a}}\end{array}$ & $\begin{array}{l}\text { Surgery dominated all other } \\
\text { options. ADA dominated IFX }\end{array}$ \\
\hline $\begin{array}{l}\text { Essat et al., } 2016 \\
{[25]^{c}}\end{array}$ & NA & NA & $\begin{array}{l}\text { Surgery is an option: VED vs. S-S } \\
\text { dominated VED } \\
\text { Surgery is not an option, mixed ITT } \\
\text { population: VED vs. SC }=£ 53,084 \\
\text { Surgery is not an option, anti-TNF } \alpha \text { failure } \\
\text { population: VED vs. SC }=£ 48,205\end{array}$ & $\begin{array}{l}\text { When surgery is an option it } \\
\text { dominated VED. When } \\
\text { surgery is not an option, VED } \\
\text { was not cost-effective } \\
\text { compared with SC }\end{array}$ \\
\hline
\end{tabular}

CAN\$1 = US\$0.794; $€ 1=$ US\$1.184; £1 = US\$1.324; 1 PLN $=$ US\$0.278

IFX infliximab, ADA adalimumab, GOL golimumab, GOL 50 golimumab $50 \mathrm{mg}, G O L 100$ golimumab $100 \mathrm{mg}$, VED vedolizumab, SC standard/ usual care/conventional treatment, $S$ surgery, $C S P$ cyclosporine, $Q A L Y G$ quality-adjusted life-years gained, ICER incremental cost-effectiveness ratio, $N A$ not available, $T N F$ tumor necrosis factor

${ }^{a}$ Our own calculations based on available data

${ }^{b}$ Results based on data from clinical studies

${ }^{c}$ Results of the ERG's own calculations were included

Essat et al. [25] was not assessed as it presents the results of a Health Technology Assessment (HTA) rather than typical economic analysis.

The identified economic analyses obtained a rather high average result of the ISPOR CHEERS statement checklist, equalling 21 points, with a minimum and maximum value of 14 and 23, respectively. Only one study obtained the minimum value [19], whereas the maximum value was reached by three studies [14, 22, 24]. The most common value was the average value, i.e. 21 points (see electronic supplementary material).

All the included studies had adequate titles, which identified the study as an economic evaluation (the most common phrases were cost-effectiveness, cost-utility, economic evaluation) and described the compared interventions. Studies failed to report relevant aspects on the system in which the decision needed to be made-adequate information was presented in only six studies $(40 \%)$ 
Table 3 The results of quality assessment of included studies with ISPOR CHEERS statement checklist

\begin{tabular}{|c|c|c|c|c|}
\hline Section/item & $\begin{array}{l}\text { Item } \\
\text { no. }\end{array}$ & Recommendation & $\begin{array}{l}\text { Number of studies } \\
\text { in line with } \\
\text { recommendation }\end{array}$ & $\begin{array}{l}\text { Percentage of studies } \\
\text { in line with } \\
\text { recommendation }\end{array}$ \\
\hline \multicolumn{5}{|l|}{ Title and abstract } \\
\hline Title & 1 & $\begin{array}{l}\text { Identify the study as an economic evaluation, or use more } \\
\text { specific terms such as "cost-effectiveness analysis" and } \\
\text { describe the interventions compared }\end{array}$ & 15 & 100.0 \\
\hline Abstract & 2 & $\begin{array}{l}\text { Provide a structured summary of objectives, perspective, } \\
\text { setting, methods (including study design and inputs), } \\
\text { results (including base-case and uncertainty analyses), } \\
\text { and conclusions }\end{array}$ & 15 & 100.0 \\
\hline \multicolumn{5}{|l|}{ Introduction } \\
\hline Background and objectives & 3 & $\begin{array}{l}\text { Provide an explicit statement of the broader context for the } \\
\text { study. Present the study question and its relevance for } \\
\text { health policy or practice decisions }\end{array}$ & 15 & 100.0 \\
\hline \multicolumn{5}{|l|}{ Methods } \\
\hline $\begin{array}{l}\text { Target population and } \\
\text { subgroups }\end{array}$ & 4 & $\begin{array}{l}\text { Describe characteristics of the base-case population and } \\
\text { subgroups analyzed including why they were chosen }\end{array}$ & 15 & 100.0 \\
\hline Setting and location & 5 & $\begin{array}{l}\text { State relevant aspects of the system(s) in which the } \\
\text { decision }(\mathrm{s}) \text { need(s) to be made }\end{array}$ & 6 & 40.0 \\
\hline Study perspective & 6 & $\begin{array}{l}\text { Describe the perspective of the study and relate this to the } \\
\text { costs being evaluated }\end{array}$ & 14 & 93.3 \\
\hline Comparators & 7 & $\begin{array}{l}\text { Describe the interventions or strategies being compared } \\
\text { and state why they were chosen }\end{array}$ & 15 & 100.0 \\
\hline Time horizon & 8 & $\begin{array}{l}\text { State the time horizon(s) over which costs and } \\
\text { consequences are being evaluated and say why } \\
\text { appropriate }\end{array}$ & 15 & 100.0 \\
\hline Discount rate & 9 & $\begin{array}{l}\text { Report the choice of discount rate(s) used for costs and } \\
\text { outcomes and say why appropriate }\end{array}$ & 13 & 86.7 \\
\hline Choice of health outcomes & 10 & $\begin{array}{l}\text { Describe what outcomes were used as the measure(s) of } \\
\text { benefit in the evaluation and their relevance for the type } \\
\text { of analysis performed }\end{array}$ & 15 & 100.0 \\
\hline \multirow[t]{2}{*}{$\begin{array}{l}\text { Measurement of } \\
\text { effectiveness }\end{array}$} & $11 \mathrm{a}$ & $\begin{array}{l}\text { Single study-based estimates: Describe fully the design } \\
\text { features of the single effectiveness study and why the } \\
\text { single study was a sufficient source of clinical } \\
\text { effectiveness data }\end{array}$ & 14 & 93.3 \\
\hline & $11 \mathrm{~b}$ & $\begin{array}{l}\text { Synthesis-based estimates: Describe fully the methods used } \\
\text { for the identification of included studies and synthesis of } \\
\text { clinical effectiveness data }\end{array}$ & & \\
\hline $\begin{array}{l}\text { Measurement and } \\
\text { valuation of preference- } \\
\text { based outcomes }\end{array}$ & 12 & $\begin{array}{l}\text { If applicable, describe the population and methods used to } \\
\text { elicit preferences for outcomes }\end{array}$ & 13 & 86.7 \\
\hline \multirow[t]{2}{*}{$\begin{array}{l}\text { Estimating resources and } \\
\text { costs }\end{array}$} & $13 \mathrm{a}$ & $\begin{array}{l}\text { Single study-based economic evaluation: Describe } \\
\text { approaches used to estimate resource use associated with } \\
\text { the alternative interventions. Describe primary or } \\
\text { secondary research methods for valuing each resource } \\
\text { item in terms of its unit cost. Describe any adjustments } \\
\text { made to approximate to opportunity costs }\end{array}$ & 15 & 100.0 \\
\hline & $13 b$ & $\begin{array}{l}\text { Model-based economic evaluation: Describe approaches } \\
\text { and data sources used to estimate resource use associated } \\
\text { with model health states. Describe primary or secondary } \\
\text { research methods for valuing each resource item in terms } \\
\text { of its unit cost. Describe any adjustments made to } \\
\text { approximate to opportunity costs }\end{array}$ & & \\
\hline
\end{tabular}


Table 3 continued

\begin{tabular}{|c|c|c|c|c|}
\hline Section/item & $\begin{array}{l}\text { Item } \\
\text { no. }\end{array}$ & Recommendation & $\begin{array}{l}\text { Number of studies } \\
\text { in line with } \\
\text { recommendation }\end{array}$ & $\begin{array}{l}\text { Percentage of studies } \\
\text { in line with } \\
\text { recommendation }\end{array}$ \\
\hline $\begin{array}{l}\text { Currency, price date, and } \\
\text { conversion }\end{array}$ & 14 & $\begin{array}{l}\text { Report the dates of the estimated resource quantities and } \\
\text { unit costs. Describe methods for adjusting estimated unit } \\
\text { costs to the year of reported costs if necessary. Describe } \\
\text { methods for converting costs into a common currency } \\
\text { base and the exchange rate }\end{array}$ & 13 & 86.7 \\
\hline Choice of model & 15 & $\begin{array}{l}\text { Describe and give reasons for the specific type of decision- } \\
\text { analytic model used. Providing a figure to show model } \\
\text { structure is strongly recommended }\end{array}$ & 14 & 93.3 \\
\hline Assumptions & 16 & $\begin{array}{l}\text { Describe all structural or other assumptions underpinning } \\
\text { the decision-analytic model }\end{array}$ & 13 & 86.7 \\
\hline Analytic methods & 17 & $\begin{array}{l}\text { Describe all analytic methods supporting the evaluation. } \\
\text { This could include methods for dealing with skewed, } \\
\text { missing, or censored data; extrapolation methods; } \\
\text { methods for pooling data; approaches to validate or make } \\
\text { adjustments (e.g., half-cycle corrections) to a model; and } \\
\text { methods for handling population heterogeneity and } \\
\text { uncertainty }\end{array}$ & 12 & 80.0 \\
\hline \multicolumn{5}{|l|}{ Results } \\
\hline Study parameters & 18 & $\begin{array}{l}\text { Report the values, ranges, references, and if used, } \\
\text { probability distributions for all parameters. Report } \\
\text { reasons or sources for distributions used to represent } \\
\text { uncertainty where appropriate. Providing a table to show } \\
\text { the input values is strongly recommended }\end{array}$ & 14 & 93.3 \\
\hline $\begin{array}{l}\text { Incremental costs and } \\
\text { outcomes }\end{array}$ & 19 & $\begin{array}{l}\text { For each intervention, report mean values for the main } \\
\text { categories of estimated costs and outcomes of interest, as } \\
\text { well as mean differences between the comparator groups. } \\
\text { If applicable, report incremental cost-effectiveness ratios }\end{array}$ & 15 & 100.0 \\
\hline \multirow[t]{2}{*}{ Characterizing uncertainty } & $20 \mathrm{a}$ & $\begin{array}{l}\text { Single study-based economic evaluation: Describe the } \\
\text { effects of sampling uncertainty for estimated incremental } \\
\text { cost, incremental effectiveness, and incremental cost- } \\
\text { effectiveness, together with the impact of methodological } \\
\text { assumptions (such as discount rate, study perspective) }\end{array}$ & & \\
\hline & $20 \mathrm{~b}$ & $\begin{array}{l}\text { Model-based economic evaluation: Describe the effects on } \\
\text { the results of uncertainty for all input parameters, and } \\
\text { uncertainty related to the structure of the model and } \\
\text { assumptions }\end{array}$ & 14 & 93.3 \\
\hline $\begin{array}{l}\text { Characterizing } \\
\text { heterogeneity }\end{array}$ & 21 & $\begin{array}{l}\text { If applicable, report differences in costs, outcomes, or cost- } \\
\text { effectiveness that can be explained by variations between } \\
\text { subgroups of patients with different baseline } \\
\text { characteristics or other observed variability in effects that } \\
\text { are not reducible by more information }\end{array}$ & 0 & 0.0 \\
\hline \multicolumn{5}{|l|}{ Discussion } \\
\hline $\begin{array}{l}\text { Study findings, limitations, } \\
\text { generalizability, and } \\
\text { current knowledge }\end{array}$ & 22 & $\begin{array}{l}\text { Summarize key study findings and describe how they } \\
\text { support the conclusions reached. Discuss limitations and } \\
\text { the generalizability of the findings and how the findings } \\
\text { fit with current knowledge }\end{array}$ & 13 & 86.7 \\
\hline \multicolumn{5}{|l|}{ Other } \\
\hline Source of funding & 23 & $\begin{array}{l}\text { Describe how the study was funded and the role of the } \\
\text { funder in the identification, design, conduct, and } \\
\text { reporting of the analysis. Describe other nonmonetary } \\
\text { sources of support }\end{array}$ & 13 & 86.7 \\
\hline Conflicts of interest & 24 & $\begin{array}{l}\text { Describe any potential for conflict of interest among study } \\
\text { contributors in accordance with journal policy. In the } \\
\text { absence of a journal policy, we recommend authors } \\
\text { comply with International Committee of Medical Journal } \\
\text { Editors' recommendations }\end{array}$ & 12 & 80.0 \\
\hline
\end{tabular}


$[14,16,18,21,22,24]$. The healthcare system and reimbursement status of the drugs analysed in the particular countries were rarely described. Study perspective was rather properly described, only in one study was the information missing [23]. In a majority of revealed analyses [2, 12-19, 21, 22] a public payer perspective was applied, and in the case of two evaluations [22, 24] a social point of view was employed. One study [11] did not report information on discount rates for costs and outcomes, but the time horizon of this study was short (1 year) and discounting was not needed. In another study [19], there was no information regarding the discount rate for outcomes, and only the rate for costs was reported. Preferences for outcomes (utility weights) were included and properly described in 13 studies (87\%), whereas in the studies by Toor et al. [10] and Yokomizo et al. [11] they were not included because they employed a different type of analysis (cost-effectiveness, with remission and response as outcomes). In some studies, information on the currency and/or exchange rates was missing. Beilman et al. [23] did not describe the year of reported costs, and Xie et al. [18] presented 2008 costs in Canadian dollars but did not present the exchange rate.

Only one study [10] did not reveal the values for main study parameters; the authors presented only the cost per remission and cost per response for biologics versus conventional therapy and other biologics. The heterogeneity was not assessed in any of the identified studies as no study included subgroups.

\subsection{Drivers of Cost-Effectiveness}

\subsubsection{Uncertainty Within the Study}

In 14 studies (88\%), sensitivity analyses were carried out, and only in the study by Moradi et al. [19] were the effects of sampling uncertainty for estimated results not described as the authors failed to undertake the probabilistic sensitivity analysis. In the study by Essat et al. [25], where the results of HTA were presented, no information on sensitivity analysis was available. Both deterministic and probabilistic sensitivity analyses were performed, and one-way sensitivity analysis was the most often used type of deterministic sensitivity analyses. The most common sensitive variables included in the analyses were the parameters of efficacy (remission/response rates, transition probabilities), utility weights, discount rates, cost parameters, and patients' characteristics (weight). The majority of studies revealed that the change of utility weights $[12,13,16-18,20-24]$ and clinical parameters, i.e. remission/response rates, transition probabilities, $[13,14,17,18,21,22,24]$ had the highest influence on analysis results (ICER/ICUR values). The change of patients' characteristics. i.e. body weight, [12, 13] also had a significant influence on the results in some cases. The costs were less often included in the sensitivity analysis; however, they had a significant influence on the results in a few cases, i.e. the cost of response to adalimumab treatment [23], infliximab cost [20], health-state costs [14], and drug costs [11]. In two studies, the change of the duration of treatment had a great influence on the results of the analysis [10, 24]. The variable related to surgery, i.e. the probability of surgical complications, was the least sensitive [20, 21, 23, 24].

\subsubsection{Uncertainty Between Studies}

The results of some studies were also compared to estimate the impact of model assumptions on the results of the analysis. We chose analyses with the same outcomes (i.e. QALY), the same comparator (i.e. standard care) and the same intervention (i.e. infliximab, as the highest number of studies related to this biologic). Seven studies met the above criteria [15-21], but two studies had to be subsequently excluded as one included different treatment strategies (infliximab $5 \mathrm{mg} / \mathrm{kg}+$ infliximab $\quad 10 \mathrm{mg} / \mathrm{kg}+$ adalimumab, and infliximab + adalimumab [18]), and one provided results separately for responders and patients in remission [15]. The range of the ICUR value in the remaining studies ranged from US\$89,468 [16, 17] to US\$240,903 [19]; however, the conclusions relating to cost-effectiveness were the same: infliximab was not a cost-effective option in the treatment of UC compared with standard care alone. All analyses used the Markov modelling, but the time horizon differed significantly from 5 years [19] to lifetime [16], and the lowest and highest ICUR values were obtained for analyses with the longest and shortest time horizon, respectively. The other model assumptions were similar-discount rates: $5 \%$ in the majority of cases, or $3.5 \%$ in the studies by Tappenden et al. [17], Archer et al. [16] and Stawowczyk et al. [21] (only for effects); study perspective: public payer/health system; reference year: 2013-2015; utility weights: based on the studies by Arseneau et al. [26] (all studies) and Woehl et al. [27] (studies [16, 17, 21]).

\subsection{Model Structure}

To compare the structures of the models, we first chose analyses that used the Markov modelling as this was the methodology most often performed; we identified 12 such analyses [14-25], but in study by Essat et al. [25] the model structure was not presented in a form of diagram and no 
detailed information was provided. That was the reason why this study was excluded.

Beilman et al. [23] and Ung et al. [20] compared two scenarios-when adalimumab is available and when it is not available. Several health states were identified: unwell during treatment, response (to pharmacotherapy or surgery), complications (after pharmacotherapy or surgery) and death. Chronic pouchitis was analysed regardless of complications after surgery and was considered as a separate state.

The structure of the model presented by Moradi et al. [19] was less sophisticated and included only three states, i.e. remission, response and surgery.

All three models used in the studies by Stawowczyk et al. [21, 22, 24] were similar and included the following states: remission (after pharmacotherapy or surgery), response, active disease, surgery, and complications after surgery. The model for infliximab [21] additionally included the second-line treatment with adalimumab (after failure of infliximab treatment).

Similar states and model structures were included in the study by Tsai et al. [15], Archer et al. [16] and Tappenden et al. [17]; however, only first-line biologic treatment was included. Tsai et al. [15] included the following health states: temporary discontinuers, remission, mild, moderate-severe, surgery, post-surgery remission and post-surgery complications. Archer et al. [16] and Tappenden et al. [17] divided the model structure into four parts, i.e. on biological treatment, non-biological treatment, post-surgery, and dead. The first and second parts included the same states: active UC, response, and remission, while the third and fourth parts included only one state: post-surgery (with/without complications) and dead, respectively.

Wilson et al. [14] did not include the response state, but instead two others were included: mild with a Mayo score of 3-5 and moderate-severe with a Mayo score of 6-12. Additionally, six other states were included: remission with a Mayo score of 0-2, surgery (from moderate-severe state), post-surgery remission, post-surgery complications, discontinue and death.

In the analysis by Xie et al. [18], three strategies were included: usual care, infliximab $5 \mathrm{mg} / \mathrm{kg}$ followed by adalimumab, and infliximab $5 \mathrm{mg} / \mathrm{kg}$ followed by infliximab $10 \mathrm{mg} / \mathrm{kg}$ and then adalimumab. The states in the model were similar to those in other studies, i.e. response, remission, surgery, complications, and active disease.

We also compared the model structures in analyses where the decision trees were the chosen methods of modelling [11-14]; a significant heterogeneity in the approaches used in the considered analyses was revealed. The structure of the decision trees and the assumptions included in the modelling varied among the studies.

Chaudhary and Fan [12] used the decision tree to compare infliximab, cyclosporine and surgery over a 1-year time horizon. The following states were included: surgery or remission after pharmacotherapy, and remission or complication after surgery.

In another study, infliximab treatment was compared with standard care, cyclosporine and surgery [13]. The states included initial remission and early surgery, and initial remission transitioned to ongoing remission and late surgery states.

Quite a simple version of the decision tree was used in the study by Wilson et al. [14] for the evaluation of biologic induction. Patients who responded to therapy during the induction period and did not discontinue as a result of adverse effects, remained on maintenance treatment (entered Markov model). Patients who did not respond during the induction phase, lost response during the maintenance phase, or discontinued due to adverse events switched to induction with conventional therapy [14].

No detailed structure of the decision analytical model was provided in the case of the decision tree used to compare the cost-effectiveness of the first-line treatment with adalimumab, infliximab or vedolizumab to induce mucosal healing [11]. The authors presented only a simplified scheme, without particular states or transitions. The lack of data on the methods used makes it impossible to compare the model structure with other analyses.

\subsection{Data Sources}

The half of analyses included the results of single clinical trials collected in the review of literature in order to estimate the transition probabilities in the model [10-12, 15, 19, 22-24]. In other studies, the meta-analyses and indirect comparisons (network meta-analyses) of the identified clinical trials were conducted to obtain the clinical data used as input for simulations [13, 14, 16-18, 20, 21, 25].

The country databases were a source of information to estimate the unit costs of drugs and medical services in 13 of 16 identified analyses [10-19, 21, 22, 24]. In only two studies [20, 23] was the literature search conducted to obtain the cost data. In study by Essat et al., no information on cost sources was provided [25].

\section{Discussion}

We identified 16 economic analyses, mainly cost-utility analyses, which assessed the cost-effectiveness of biologic drugs (infliximab, adalimumab, golimumab, and vedolizu$\mathrm{mab}$ ) in the treatment of UC. The assessed interventions were mainly compared with standard care alone, but cyclosporine, surgical intervention and other biologics were also included as comparators in some cases. The highest 
number of economic analyses related to infliximab, and only three of the analyses included vedolizumab, including one HTA report. The vast majority of studies used Markov modelling and only five studies used the decision trees. All Markov models included response, remission, and surgery states, but response state was, in some cases, replaced by mild state. The second-line biologic treatment was rarely included in the analysis. The structure of decision trees varied significantly between the studies. The most commonly applied time horizon was lifetime (30 years or more), which was adopted in $44 \%$ of analyses ( 7 of 16), but a 1-year time horizon was also employed quite often (27\% of the analyses). In our opinion, a lifetime horizon is more valuable for chronic diseases as the longer period of observation provide more sophisticated information on consequences of therapy compared with a 1-year horizon. The QALY was used as an outcome in $88 \%$ of the analyses, while the other analyses used remission, response and mucosal healing. Cost-effectiveness studies on new treatments should take into consideration the impact of the new interventions on patient productivity and a corresponding economic burden for the society. Therefore, it should be emphasized that only two of the economic analyses also carried out the calculations from a social perspective, and took into account the impact of treatment on work productivity, which seems quite unusual because UC, a condition usually diagnosed in middle-aged patients, generates a significant loss of productivity; therefore, indirect costs could even exceed direct healthcare costs. Both analyses were conducted in Polish settings [22, 24]. Further studies should be performed to measure the influence of productivity loss on the cost-effectiveness of biologic drugs in UC.

The quality of the included analyses was evaluated as high, based on review of methodologies used and total score of the CHEERS reporting quality. A majority of valuable and important information referring to reporting quality was provided in the reports collected, excluding the description of national healthcare systems or reimbursement status of assessed intervention, which was quite rarely presented in the analyses.

According to reimbursement criteria, the acceptable cost-effectiveness of biologics compared with standard care alone was revealed in only three studies. The performed sensitivity analyses demonstrated that the costeffectiveness results (ICER/ICUR values) were the most sensitive to the change of utility weights and clinical parameters (response/remission rates, transition probabilities). The between-study comparison may suggest that the longer the time horizon, the lower the ICUR values. This may result from the fact that UC is a lifelong disease and its effects revealed during the whole lifespan.

The included analyses were country-specific as they included the costs of therapies valid for each specified country. Clinical outcomes can be transferred to other countries and generalized; however, cost inputs are largely country-specific, which in turn limits the transferability and generalizability of the results and conclusions to other countries. Another issue is the differences in healthcare systems and reimbursement policies between countries, and also the methods of inpatient or outpatient care, which may have a significant influence on the results and final conclusions of economic evaluations.

We critically reviewed the collected publications but also discussed the model structures, cost sources, model assumptions, model validation and key drivers for costeffectiveness analyses to provide a substantial contribution over the existing reviews in the topic.

We limited the final review to studies written in English, which may be considered as a limitation, as this approach produced the potential omission of relevant papers. Several identified analysis were conducted directly by manufacturers and their results were published in a form of an HTA report. We did not include the results of manufacturers' calculations, which may also be a limitation, but the results of analysis carried out by HTA agencies were taken into account. Such an approach was implemented because manufacturers' analysis, which are industry-sponsored evaluations, may be biased. Another limitation is that the quality assessment was not performed for one analysis [25]. The analysis results were presented as an ERG assessment and did not include the structure typical for economic evaluations. The heterogeneity of model assumptions was the reason why meta-analysis was not performed for identified studies, which also constitutes a limitation of this review.

Despite the above limitations, we included the majority of cost-effectiveness studies currently available, which enabled us to evaluate the overall quality and identify key drivers of cost-effectiveness.

This study is the most comprehensive review that incorporates economic evaluations referring to UC treatments. It includes quality assessment with a validated instrument and in-depth analysis of cost-effectiveness key drivers.

There is a need for studies that will provide evidence for the effectiveness of long-term treatment of UC and resolve the uncertainty associated with the key drivers of efficiency.

\section{Conclusions}

We identified well-reported economic analyses providing conclusions on the cost-effectiveness of infliximab, adalimumab, golimumab and vedolizumab therapy in UC. Conclusions based on the performed cost-effectiveness analyses were not unequivocal. The key drivers identified in the review were utility weights and clinical inputs. 
Data Availability Statement We made every effort to provide a sophisticated and complex set of data while performing the review. Any data valid during the current review were presented in the electronic upplementary material.

Authors Contributions ES and PK contributed to the study rationale and design, and performed a systematic review and quality assessment of identified studies. ES carried out interpretation of the data. Both authors prepared the draft of the manuscript, contributed to editing and revising the manuscript for important intellectual contributions, and approved the final version submitted for publication.

Funding No specific funding was used to carry out this review or prepare this article.

\section{Compliance with Ethical Standards}

Conflict of interest Ewa Stawowczyk and Paweł Kawalec declare that they have no conflicts of interest.

Open Access This article is distributed under the terms of the Creative Commons Attribution-NonCommercial 4.0 International License (http://creativecommons.org/licenses/by-nc/4.0/), which permits any noncommercial use, distribution, and reproduction in any medium, provided you give appropriate credit to the original author(s) and the source, provide a link to the Creative Commons license, and indicate if changes were made.

\section{References}

1. Baumgart DC, Sandborn WJ. Inflammatory bowel disease: clinical aspects and established and evolving therapies. Lancet. 2007;369:1641-57.

2. Kornbluth A, Sachar DB. The Practice Parameters Committee of the American College of Gastroenterology. Ulcerative Colitis Practice Guidelines in Adults: American College of Gastroenterology, Practice Parameters Committee. Am J Gastroenterol. 2010;105:501-23.

3. Ordás I, Eckmann L, Talamini M, et al. Ulcerative colitis. Lancet. 2012;380:1606-19.

4. Ananthakrishnan AN. Epidemiology and risk factors for IBD. Nat Rev Gastroenterol Hepatol. 2015;12:205-17.

5. Aggarwal A, Sabol T, Vaziri H. Update on the use of biologic therapy in ulcerative colitis. Curr Treat Options Gastroenterol. 2017;15:155-67.

6. Stone $\mathrm{CD}$. The economic burden of inflammatory bowel disease: clear problem, unclear solution. Dig Dis Sci. 2012;57(12):3042-4.

7. M'Koma AE. Inflammatory bowel disease: an expanding global health problem. Clin Med Insights Gastroenterol. 2013;6:33-47.

8. Higgins JPT, Green S, editors. Cochrane handbook for systematic reviews of interventions. http://handbook.cochrane.org. Accessed July 2017

9. Husereau D, Drummond M, Petrou S, et al. Consolidated Health Economic Evaluation Reporting Standards (CHEERS)—Explanation and Elaboration: a report of the ISPOR Health Economic Evaluation Publication Guidelines Good Reporting Practices Task Force. Value Health. 2013;16:231-50.

10. Toor K, Druyts E, Jansen JP, Thorlund K. Cost per remission and cost per response with infliximab, adalimumab, and golimumab for the treatment of moderately-to-severely active ulcerative colitis. J Med Econ. 2015;18(6):437-46.

11. Yokomizo L, Limketkai B, Park KT. Cost-effectiveness of adalimumab, infliximab or vedolizumab as first-line biological therapy in moderate-to-severe ulcerative colitis. BMJ Open Gastroenterol. 2016;3(1):e000093.

12. Chaudhary MA, Fan T. Cost-effectiveness of infliximab for the treatment of acute exacerbations of ulcerative colitis in the Netherlands. Biol Ther. 2013;3:45-60.

13. Punekar YS, Hawkins N. Cost-effectiveness of infliximab for the treatment of acute exacerbations of ulcerative colitis. Eur J Health Econ. 2010;11:67-76.

14. Wilson MR, Bergman A, Chevrou-Severac H, Selby R, Smyth M, Kerrigan MC. Cost-effectiveness of vedolizumab compared with infliximab, adalimumab, and golimumab in patients with ulcerative colitis in the United Kingdom. Eur J Health Econ. 2017. https://doi.org/10.1007/s10198-017-0879-5.

15. Tsai HH, Punekar YS, Morris J, Fortun P. A model of the longterm cost effectiveness of scheduled maintenance treatment with infliximab for moderate-to-severe ulcerative colitis. Aliment Pharmacol Ther. 2008;28(10):1230-9.

16. Archer R, Tappenden $P$, Ren $S$, et al. Infliximab, adalimumab and golimumab for treating moderately to severely active ulcerative colitis after the failure of conventional therapy (including a review of TA140 and TA262): clinical effectiveness systematic review and economic model. Health Technol Assess. 2016;20(39):1-326.

17. Tappenden P, Ren S, Archer R, et al. A model-based economic evaluation of biologic and non-biologic options for the treatment of adults with moderately-to-severely active ulcerative colitis after the failure of conventional therapy. PharmacoEconomics. 2016;34:1023-38.

18. Xie F, Blackhouse G, Assasi N, et al. Cost-utility analysis of infliximab and adalimumab for refractory ulcerative colitis. Cost Eff Resour Alloc. 2009;11:7-20.

19. Moradi N, Tofighi S, Zanganeh M, et al. Economic evaluation of infliximab for treatment of refractory ulcerative colitis in Iran: cost-effectiveness analysis. Iran J Pharm Sci. 2016;12(4):33-42.

20. Ung V, Thanh NX, Wong K, et al. Real-life treatment paradigms show infliximab is cost-effective for management of ulcerative colitis. Clin Gastroenterol Hepatol. 2014;12:1871-8.

21. Stawowczyk E, Kawalec P, Pilc A. Cost-utility analysis of infliximab with standard care versus standard care alone for induction and maintenance treatment of patients with ulcerative colitis in Poland. Pharmacotherapy. 2016;36(5):472-81.

22. Stawowczyk E, Kawalec P, Pilc A. Cost-utility analysis of 1-year treatment with adalimumab/standard care and standard care alone for ulcerative colitis in Poland. Eur $\mathrm{J}$ Clin Pharmacol. 2016;72:1319-25.

23. Beilman CL, Thanh NX, Ung V, et al. Real-life treatment paradigms show adalimumab is cost-effective for the management of ulcerative colitis. Can J Gastroenterol Hepatol. 2016;2016:5315798.

24. Stawowczyk E, Kawalec P, Pilc A. Cost-effectiveness analysis of 1-year treatment with golimumab/standard care and standard care alone for ulcerative colitis in Poland. PLoS One. 2016;11(8):e0160444.

25. Essat M, Tappenden $P$, Ren $S$, et al. Vedolizumab for the treatment of adults with moderate-to-severe active ulcerative colitis: an evidence review group perspective of a NICE single technology appraisal. Pharmacoeconomics. 2016;34(3):245-57.

26. Arseneau KO, Sultan S, Provenzale DT, et al. Do patient preferences influence decisions on treatment for patients with steroidrefractory ulcerative colitis? Clin Gastroenterol Hepatol. 2006; 4:1135-42.

27. Woehl A, Hawthorne B, Morgan CL, et al. The epidemiology and healthcare resource use in patients with Crohn's disease: a population based UK study. Value Health. 2007;10:A355. 